\title{
Comparison of Intracranial Pressure by Lateral and Frontal Impacts \\ - Validation of Computational Model
}

\author{
Aalap Patel and Tarun Goswami
}

Additional information is available at the end of the chapter

http://dx.doi.org/10.5772/50368

\section{Introduction}

Traumatic brain injury (TBI) is a leading cause of death in the United States. The brain is among the most essential organs of the human body. From a mechanical stand point, different scenarios where a head comes in contact with a media has evolved a number of integrated protection devices. The scalp and skull, but also to a certain extent the pressurized subarchnoidal space and the dura matter, are the natural protections for the brain. However, these structures are not adapted to the dynamic loading conditions involved in modern road and sports accidents as well as blast injury scenarios. The consequence of this extreme loading is often moderate-to-severe TBI [1-15]. Injuries to the head constitute one of the major causes of death. Brain injury disables or kills someone in the United States every two and half minutes [2]. The annual hospitalization and rehabilitation cost has been estimated to be $\$ 33$ billion per year in the US alone [14]. In the United States TBI is a leading cause of death for persons under age 45 [15]. TBI occurs every 15 seconds, see Figure 1. Approximately 5 million Americans currently suffer some form of TBI disability. The leading causes of TBI are motor vehicle accidents, falls, sports injuries and from blast injuries [12]. Thus, preventing these head injuries will not only enhance safety and quality of life but also save healthcare dollars.

Over the last 40 years, biomechanical research has been gaining attention to fully understand the mechanism of the head injury. Understanding and thus protecting the brain from injury. This can only be achieved by: 1) understanding mechanics of the impact and 2) the biomechanical response of the head to a variety of the loading conditions [2]. A costeffective alternative method using the finite element modeling was used to investigate TBI of human head subject to impact loadings [3].

A brief review of TBI performed below and injury parameters compiled for model validation. 


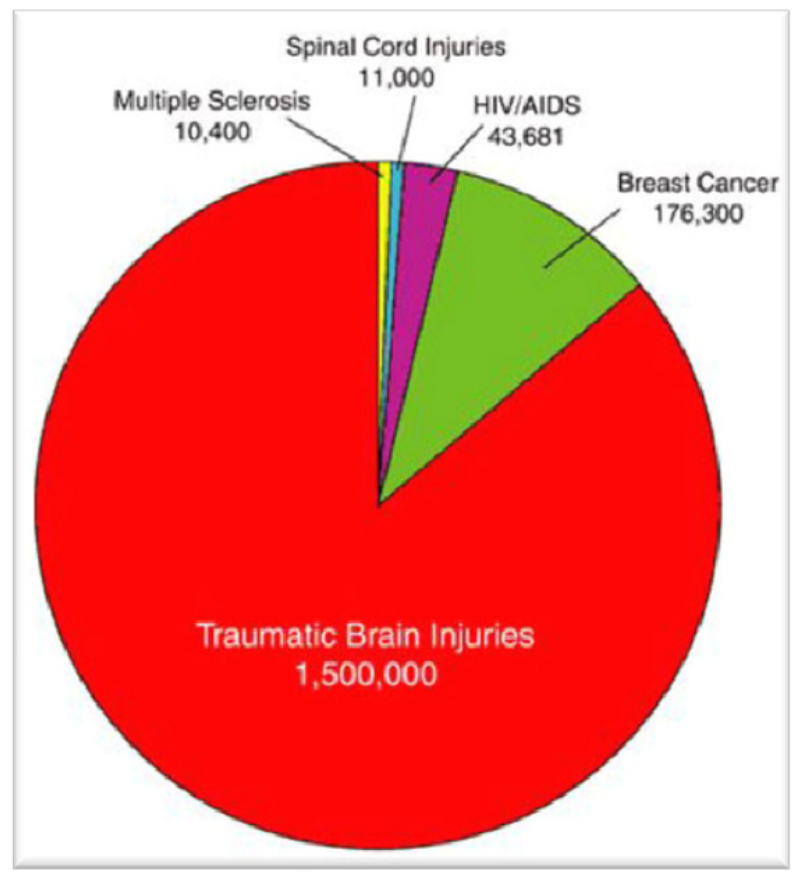

Figure 1. Scope of traumatic brain injury in comparison to other modalities [13]

\section{Head injuries}

The human head consists of three components [9],

1. The bony skull - Cranial and facial bones

2. The skin and other soft tissue covering the skull. Which consists of layers known as the SCALP (Skin, Connective Tissue, Aponeurosis (Galea), Loose connective tissue and Periosteum

3. The contents of the skull. Most notably the brain, but also including the brain's protective membranes (meninges) and numerous blood vessels, shown in Figure 2

Injuries to the skin may be categorized as superficial or deep, and include contusion (bruise), laceration (cut), and abrasion (scrape). Injuries to the skull may break one or more of the bones of the skull in which case the skull is said to have been fractured (broken). Two aspects of a skull fracture are 1) whether it is open, or 2) depressed [10]. Injuries to the brain and associated soft tissue are the result of either head impact or abrupt head movement (e.g., deceleration injury) or some combination of the two. Injuries may be due to the skull fracturing and being pushed inward (a depressed fracture), or from the brain impacting the interior of the skull, or from internal stressing of the brain (i.e., shear, tension and/or compression). The complexities of the head and brain systems are reflected in head injury consequences, Figure 3. 


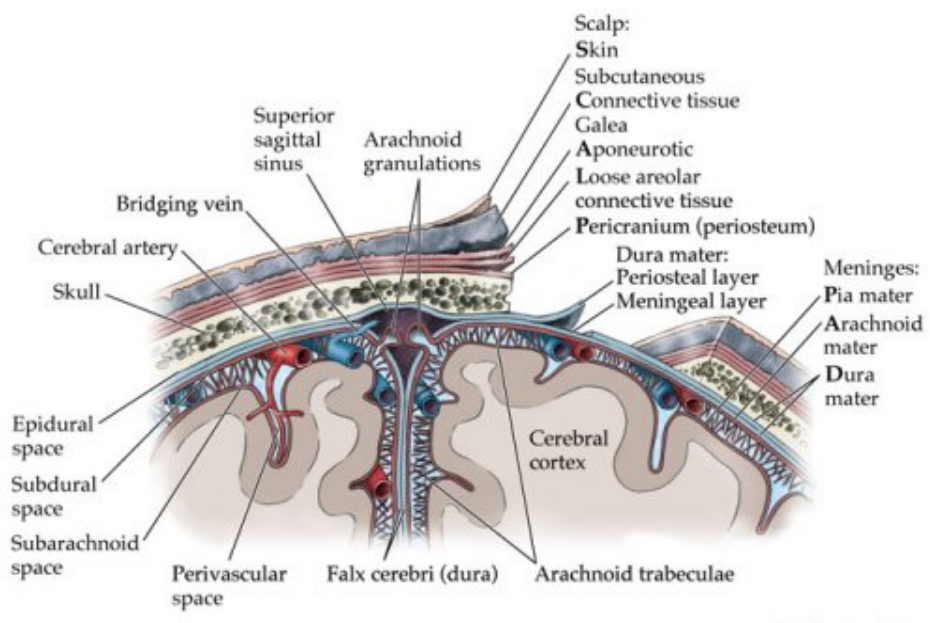

Figure 2. Anatomy of the human head [10]

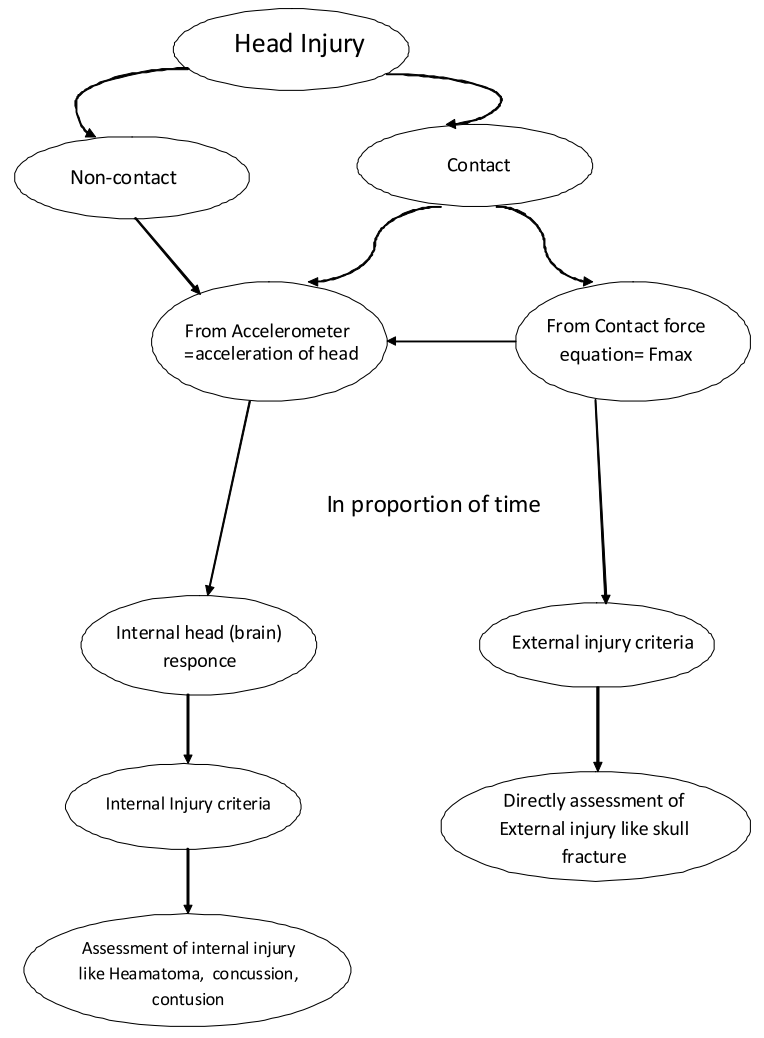

Figure 3. Flowchart of TBI injury assessment criteria development 
The injury to the brain may be categorized in terms of, 1) The cause of injury, either contact vs. non-contact, 2) The type of injury, either primary in which the injury occurs at the time of initial injury producing event, or secondary where the injury results from some injury producing event but does not develop until somewhat later (through an intermediate process such as a metabolic effect), and 3) the type of injury, either focal (i.e. fairly localized) or diffuse (rather distributed) as shown in Figure 3.

In injury producing events, there are generally 3 collisions which occur [2]:

1. The "first collision" is where injury producing event occurs, e.g. the vehicle strikes another car or object and as a result the vehicle is rapidly decelerated and/or rotated.

2. The "second collision" is the movement of the occupants in the vehicle and their subsequent contact with the vehicle interior.

3. The "third collision" is when the internal organs of the occupant collide and/or move within the occupant.

\subsection{Parameters that control head injury}

A number of publications [17-43] discuss modeling and analyses of TBI using specific tools [4445]. Gong [16] recently proposed a simple head-striker model to simulate the contact between a human head and a foreign-object striker. Based on the head-striker model, they formulated a contact force function, which is a function of time, impact mass, contact stiffness, impact velocity, and material properties of the head and neck. The contact force function was used for the estimation of the contact force between the human head and the foreign striker $[5,16]$.

\subsubsection{Force}

The contact force can be approximated [16] from the equation below. Then the estimated contact force may be used in two ways: 1) for the assessment of the exterior head injury, such as scalp damage, skull fracture, and 2) as input to the head model to predict the inner head injury, such as hematoma and brain injury [5].

$$
F \max =\frac{R *^{\frac{1}{5}} E *^{\frac{2}{5}} m *^{\frac{3}{5}} \Delta v^{\frac{6}{5}} E_{s h}^{\frac{1}{2}} h}{\left(\frac{1}{\sqrt{2.3}}\right) R *^{\frac{1}{5}} \quad E *^{\frac{2}{5}} m *^{\frac{1}{10}} \Delta v^{\frac{1}{5}} R_{s h}^{\frac{1}{2}}\left(1-v_{s h}^{2}\right)^{\frac{1}{4}}+\left(\frac{\sqrt{3}}{2}\right)\left(\frac{16}{15}\right)^{\frac{1}{10}} E_{s h}^{\frac{1}{2}} h}
$$

The terminologies are explained in the original reference [16].

$$
\frac{1}{R^{*}}=\frac{1}{R_{s o l}}+\frac{1}{R_{s h}}, \frac{1}{m^{*}}=\frac{1}{m_{s o l}}+\frac{1}{m_{s h}} \text { and } \frac{1}{E^{*}}=1-\frac{v_{s o l}^{2}}{E_{s o l}}+1-\frac{v_{s h}^{2}}{E_{s h}}
$$

\subsubsection{Time duration}

An analytical model [17] was proposed the impact of a fluid-filled spherical shell of mass $\left(\mathrm{m}_{\mathrm{sh}}\right)$, thickness $(\mathrm{h})$ and outer radius ( $\mathrm{Rsh}$ ) with a solid homogeneous isotropic elastic sphere of mass $\left(\mathrm{m}_{\mathrm{sol}}\right)$ and outer radius ( $\left.\mathrm{R}_{\mathrm{sol}}\right)$ at a relative velocity $\left(\mathrm{D}_{\mathrm{v}}\right)$ as shown in Figure 4. 


$$
T p=\pi \sqrt{\frac{\frac{3}{4}\left(\frac{16}{15}\right)^{\frac{1}{5}} m^{* \frac{4}{5}}}{R^{*} \frac{2}{5} E^{* \frac{4}{5}} \Delta v^{\frac{2}{5}}}+\frac{m^{*} R_{s h} \sqrt{\left(1-v_{s h}^{2}\right)}}{2.3 E_{s h} h^{2}}}
$$

The shell was assumed to be filled with an inviscid fluid of density ( $\rho \mathrm{f})$ and Bulk modulus (B) [5]. The impact mass, contact stiffness, impact velocity, angular velocity, accelerations, young's modulus, poison's ratio, time duration, height of the head(projectile) and an impactor influence severity of external forces were incorporated in the model. Effect of impact mass, contact stiffness and impact velocity on pressure-time histories have been described in [6].

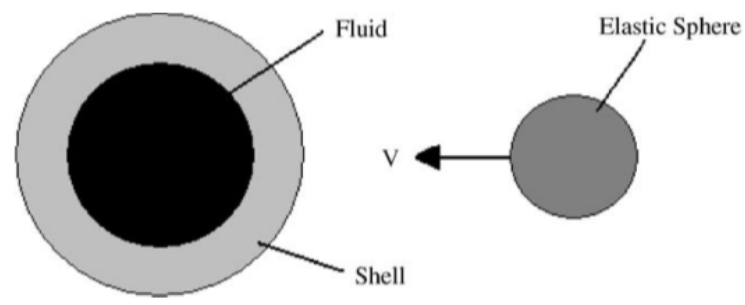

Figure 4. Illustrative representation of the analytical shell model.

(Rf- inviscid fluid of density, B- Bulk modulus, Esol; Esh and nsol; nsh are the Young's moduli and Poisson's ratio of solid and shell (Sphere), respectively. At occipital side of skull msh-mass of spherical shell-1.96 kg, $\mathrm{h}$ - thickness -0.00561 , Rsh- radius of spherical shell$0.0725 \mathrm{~m}$, Msol- mass of solid, Rsol- outer radius of solid, Dv- velocity)[5]

\subsubsection{Accelerations}

The maximum acceleration of either projectile or head, assuming a quasi-static global response of the system, can be obtained by dividing the maximum force transmitted by the mass of the projectile or head, respectively [5]. For $\mathrm{t}>6 \mathrm{~ms}$ impact time duration, neck force also needs to be considered, Figure 5, while calculating the resultant head accelerations. For short duration impacts $(<6 \mathrm{~ms})$, the neck does not influence the kinematic head response [35, 43].

\subsubsection{Contact area}

Load or force to fracture/failure of the skulls of 12 unembalmed cadavers heads were reported by Yoganandan [19]. Using a hemispherical impactor with a $48 \mathrm{~mm}$ radius, they carried out impacts to various locations on the skull at a rate of 7.1-8.0 m/s. Failure loads ranged between 8.8 and $14.1 \mathrm{kN}$, with an average of $11.9 \mathrm{kN}$. Allsop [20] carried out temporo-parietal impacts on 31 unembalmed cadaver heads with two types of flat rigid impactors-one circular and $2.54 \mathrm{~cm}$ in diameter, the other a rectangular plate $5 \times 10 \mathrm{~cm}$. Fracture force for the small circular plate ranged between 2.5 and $10 \mathrm{kN}$ with an average of $5.2 \mathrm{kN}$. Fracture force for the rectangular plate ranged between 5.8 and $17 \mathrm{kN}$ with an 


$$
\begin{aligned}
& \mathrm{m}_{\text {head }} \mathrm{a}=\mathrm{F}_{\mathrm{I}}+\mathrm{F}_{\mathrm{N}} \\
& \text { So, } \mathrm{a}=\left(\mathrm{F}_{\mathrm{I}} / \mathrm{m}_{\text {head }}\right)+\left(\mathrm{F}_{\mathrm{N}} / \mathrm{m}_{\mathrm{h}}\right.
\end{aligned}
$$

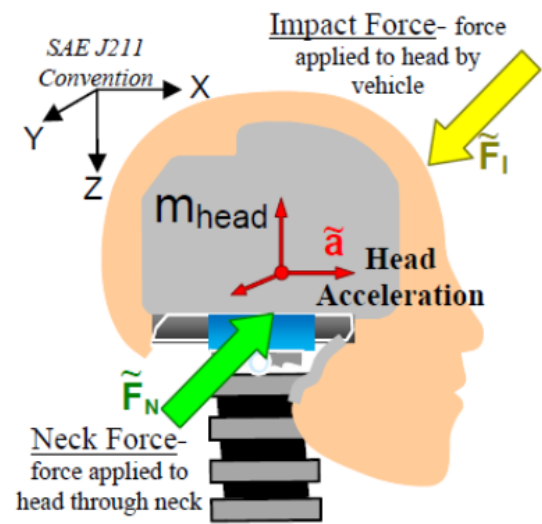

Figure 5. Force mechanism for head-neck [48]

average of $12.4 \mathrm{kN}$. The authors concluded that there is a significant relationship between contact area and fracture force. Thus, impacts with the ground are likely to require higher forces than with a smaller impactor [4].

\subsection{Head injury criteria}

Prior experiments on the capability of the human brain to hold impact forces were performed at Wayne State University using human cadavers and animal models [21, 22] as shown in Figure 6. This work led to the publication of the Wayne State Tolerance Curve [23, 24], a generally logarithmic curve that describes the relationship between the magnitude and duration of impact acceleration and the onset of skull fractures [7].

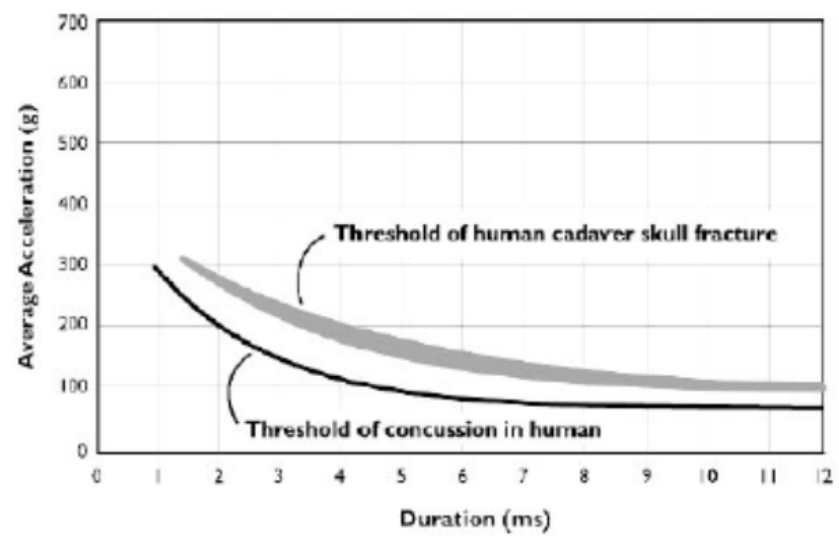

Figure 6. Wayne State Tolerance Curve $[23,24]$

The relationship is nonlinear - the head can tolerate high accelerations for very brief periods but a longer exposure to a lower acceleration level may be damaging as well, Fig. 6. For a 
given degree of injury the logarithmic slope of the exposure time and acceleration graph is approximately -2.5 . This relationship proposes the Severity Index (SI) as a measure of the injury potential of an impact [25]. SI is the integral of the acceleration time curve, weighted by the 2.5 factor observed in the Wayne State Tolerance Curve and calculated as

$$
S I=\int_{0}^{T} a^{2.5} d t
$$

Where $a(t)$ is the acceleration-time pulse of the impact and $T$ is its duration. An SI score of 1000 approximates the limit of human tolerance. Impacts with a higher score have a nonzero probability of causing a life-threatening brain trauma [7].

Severity Index SI [25] calculates distress of an impact in a way that quantifies the risk of head injury. In practice, SI scores are logical predictors of the injury potential of impacts that produce focal brain injuries. For impacts of lower intensity but longer duration, the SI calculation produces unreasonably high values that predict more severe injuries than those actually observed in cadaver experiments. The Head Injury Criterion (HIC) is an alternative measure of impact severity that is not subject to these errors. The HIC score is given by:

$$
H I C=\max \left(\left(t_{1}-t_{0}\right)\left[\frac{1}{\left(t_{1}-t_{0}\right)} \int_{t=t_{0}}^{t_{1}} a_{t} d t\right]^{2.5}\right)
$$

Where $t_{0}$ and $t_{1}$ are the beginning and end times of the portion of the acceleration-time pulse being examined. The integral account for the duration of the acceleration and an iterative search found the time interval $\left(t_{0}, t_{1}\right)$ to maximize the HIC score [7], Figure 7.
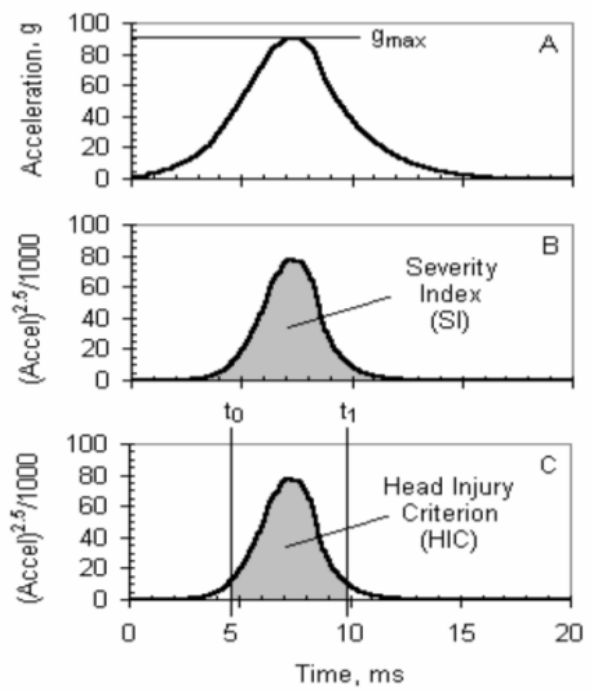

Figure 7. Example of SI and HIC calculations [7]
(A) Acceleration-time pulse from an impact between a surrogate head and an artificial turf surface, showing the peak value or gmax score.

(B) The same pulse with acceleration values exponentiated to power 2.5. The SI score is the area under the curve (C) As (B) but showing the time limits, t0 and $\mathrm{t} 1$, that maximize the HIC score. 
A HIC score of 1000 represents the "safe" limit of human tolerance, above which the risk of a serious head injury is non-zero. In the sports surfacing world, HIC scores are the primarily determinant of playground surfacing, shock attenuation performance. Other terms of surfacing shock attenuation use a $200 \mathrm{~g}$ max limiting performance criterion, on that basis it approximates the HIC limit [7].

Empirically determined relationships between HIC scores and the probability of head injury $[26,27]$ are widely used in the automotive industry to estimate the risk of injury. Figure 8 shows examples of Expanded Prasad-Mertz Curves. Each curve estimates the possibility that an impact with a given HIC score will result in a specified level of head trauma [7]. Figure 8 , also shows the relationship between the HIC score of a head impact and the probability of an injury.

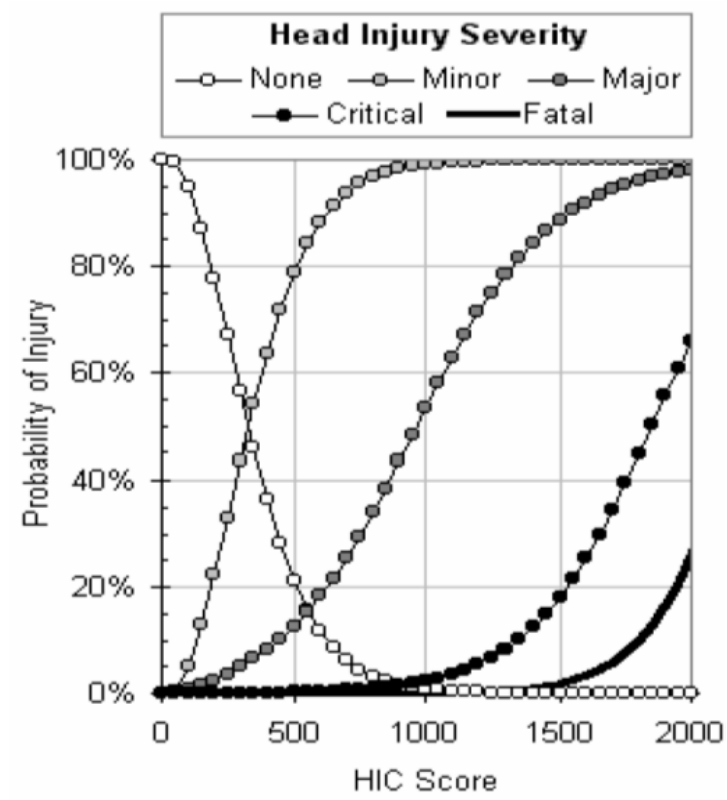

Figure 8. Expanded Prasad-Mertz Curves [7]

Computational simulation of real life head injury accidents has been used for various purposes. Some have compared AIS (abbreviated injury scale) scores for real life injuries to HIC scores or other indices of injury calculated from the reconstruction [4, 30-32]. HIC and tolerance levels have been explained [46-47] and tabulated in Table 1. Also, moderate and severe neurological injuries can only be distinguished with a criterion that is computed using intracranial variables and not with the sole global head accelerations [1]. More recently, there has been a move away from this approach of looking for a parameter that correlates well with overall severity of injury, and many are now focusing on determining tolerance limits of the head to specific lesion types, for example, acute subdural hematoma (ASDH), diffuse axonal injury (DAI) or skull fracture [4]. 


\begin{tabular}{|l|l|l|}
\hline Head Injury Criteria & AIS Code & Level Of Brain Concussion And Head Injury \\
\hline $135-519$ & 1 & Headache or dizziness \\
\hline $520-899$ & 2 & Unconscious less than 1 hour - linear fracture \\
\hline $900-1254$ & 3 & Unconscious $1-6$ hours - depressed fracture \\
\hline $1255-1574$ & 4 & Unconscious 6 - 24 hours - open fracture \\
\hline $1575-1859$ & 5 & Unconscious greater than 25 hours - large haematoma \\
\hline$>1860$ & 6 & Non survivable \\
\hline
\end{tabular}

Table 1. Levels of Consciousness In Relation To Head Injury Criteria [46]

\subsubsection{Injury criteria for Subarachnoid haematoma, contusion and skull fracture}

Tolerance curves for ASDH due to rupture of bridging veins were experimentally produced in monkeys [28] and compared with human clinical data. It was concluded that bridging veins are highly sensitive to strain-rate and tend to rupture during impacts associated with high rates of increasing acceleration. As the duration of the pulse increases, higher levels of angular acceleration will be required in order to maintain the high strain rate necessary for rupture of bridging veins. Figure 9 shows tolerance curves for rhesus monkeys. For humans, a fall resulting in head acceleration of over $200 \mathrm{~g}$ and pulse duration of $3.5 \mathrm{~ms}$ or less would create conditions necessary for the production of bridging vein ASDH [4].

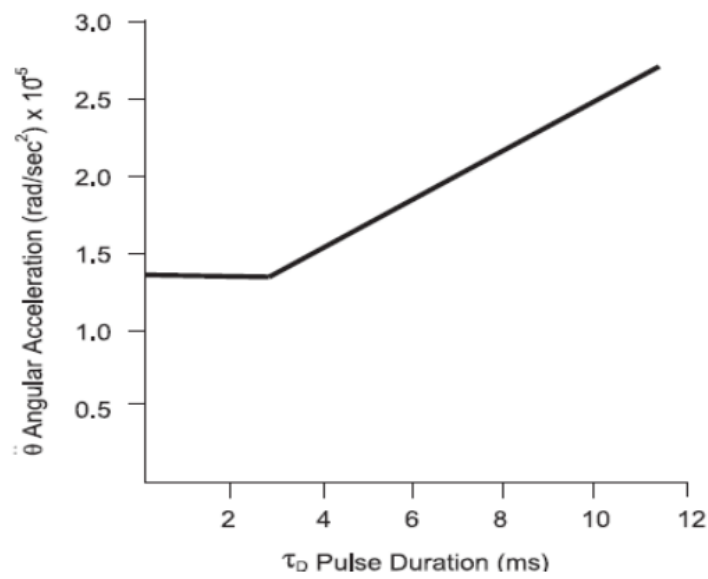

Figure 9. Injury tolerance curve for ASDH in rhesus monkeys [4]

Figure 10 shows the tolerance curves [29], derived for 5\% critical strain, below which there is no axonal injury, and $10 \%$ critical strain, below which mild injury such as concussion could be expected and above which DAI can be expected. For impacts with very stiff contacts and short durations, the brain will move relative to the skull at impact, and thus a change in angular velocity of the skull will be of prime importance and causation of injury, Fig. 10. However, for impacts with softer structures, the brain will tend to move with the head, and will thus be subjected to the same accelerations [4]. 


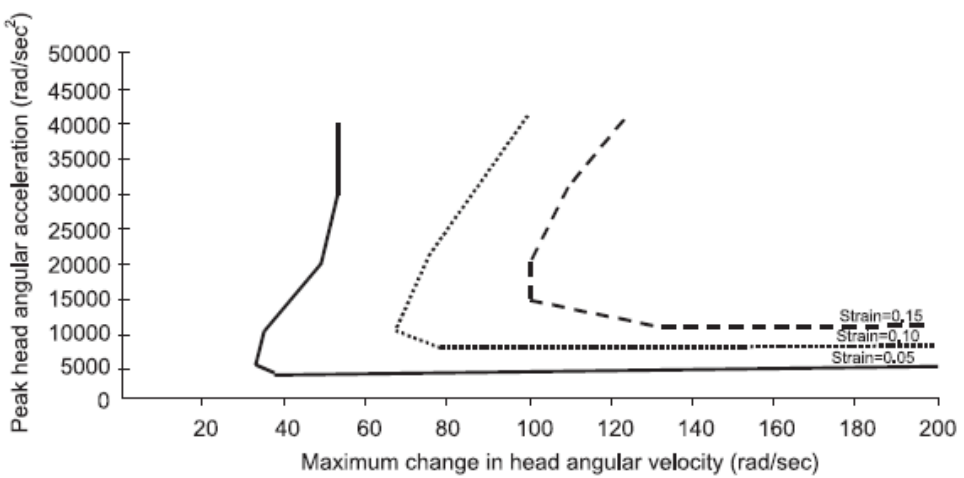

Figure 10. Tolerance curves for DAI and cerebral concussion $[4,29]$

Tolerance of the head to skull fracture is much easier to determine than tolerance to intracranial injury. This is because of the definite relationship between force applied to the skull, and failure of cranial bone. Applied maximum force can be calculated from the equations discussed in the background section of head injury. Also from the Wayne State Tolerance Curve [23-24], tolerance of the head to skull fracture can be determined [4].

Tolerance limits to specific types of head injury were from reconstructing accidents and comparing the injuries sustained with parameters calculated from the reconstructions. For example, Auer [33] reconstructed 25 fatal pedestrian accidents using various methods, including computer simulations. Head acceleration and impact duration were calculated, and from these, the upper tolerance limit (lowest level of loading above which the specific injury is always observed) and the lower tolerance limit (highest value below which the injury never occurs) for various kinds of brain injury were determined, shown in Figure 11 [4].

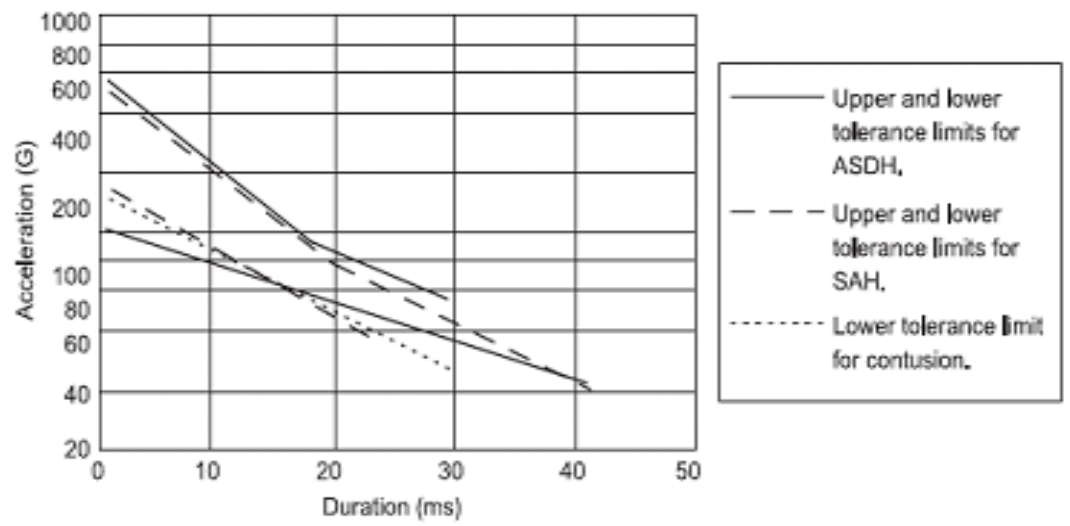

Figure 11. Upper and lower tolerance curves for ASDH, subarachnoid haematoma, and contusion [4] 
The types of lesions examined were subdural haematoma, subarachnoid haematoma and brain contusions. While the authors did not elaborate on the relationship between the mechanical parameters and the lesions observed, they concluded that reconstructing pedestrian accidents could be a useful means of estimating tolerance limits for discrete brain injuries. However, due to lack of certainty about input variables, these are still very approximate estimations [4].

Since all head injury criteria are generally explained in terms of the resultant head accelerations, the resultant head acceleration are determined by placing an accelerometer to the desired points. The impact mechanisms are related with stresses, strain and pressure induced by the impact in the head which produce injury. Besides all these parameters affecting TBI with the resultant head accelerations, the following human head injury mechanisms and tolerance limits (stress, strain and pressure) were derived from accidents reconstruction [36-37].

A brain pressure reaching $200 \mathrm{kPa}$ is an indicator for brain contusions, oedema and hematoma.

A brain Von Mises stress reaching $18 \mathrm{kPa}$ is an indicator for moderate neurological injuries. A brain Von Mises stress reaching $38 \mathrm{kPa}$ is an indicator for severe neurological injuries.

A global strain energy of the brain skull interface reaching $5.4 \mathrm{~J}$ is an indicator for subdural hematoma and subarachnoidal bleeding.

A global strain energy of the skull reaching $2.2 \mathrm{~J}$ is an indicator for skull fractures.

\section{Computational model validation}

Finite element modeling and simulation of the human head biomechanics remain scarce in the literature. Only models that exist in the literature were reported by Ruan [18], and Willinger [35] and validated with limited experimental data. As FEM of the head finds wider applications in a diversity of fields, experimental validation is a critical key element [3]. Therefore, one of the objectives of this paper was to construct a $3 \mathrm{D}$ model of the head from Magnetic Resonance Imaging and validate FE analysis with available experimental data on stress induced by frontal and lateral impacts. Two sets of experimental data were used, from Nahum [42] and [34].

Previous research used various computational software: ULP models, ScanFE/RP (Simpleware Ltd.), FEA packages MSC/PATRAN; MSC/DYTRAN; ABAQUS; LS-DYNA3D (LS-DYNA3D, LSTC), MADYMO (Mathematical Dynamic Models) - may combine both multibody and FEM techniques, Test dummy- human body models to reconstruct the accident especially vehicle/car crash, Vtk and SUDAAN (based on CT scan sets). Methodology used in this paper is discussed below.

\subsection{Methodology}

\subsubsection{Software}

MIMICS software used in this study allows user to process and edit 2D image data (CT, $\mu \mathrm{CT}$, MRI, etc.) to construct 3D models with accuracy, flexibility and user-friendliness, Figure 12. Besides smoothening, FEA, wide variety of boolean functions, the powerful 
segmentation tools allow user to segment the medical CT/MRI images, and take measurements. The designs can be modified based on the simulation outcomes and can be exported to the FEA/CFD packages [44]. Additional steps like assigning material properties, part sections, assemblies, load, boundary conditions and analysis for head models then exported into the ABAQUS [45].

\subsubsection{FE Model properties}

After exporting all four models in to ABAQUS, further simulation was done on randomly selected one of the four models. Tables 2-3 provide the subject specific dimensions and mechanical properties of the cadaver heads (computational models) used in this study. All four meshed-head models after exporting into the ABAQUS are shown in Figure 13. Further smoothening to reduce distorted elements was performed.

Skull: Total no of nodes: 51988 and total no. of elements: 210938

Brain: Total no of nodes: 36585 and total no. of elements: 145151

Young modulus, poisson's ratio and density are described in section 2.2

\begin{tabular}{|c|l|l|l|}
\hline Material & Young modulus E(Mpa) & Poisson's ratio $(v)$ & Density $\varrho\left(\mathrm{kg} / \mathrm{m}^{3}\right)$ \\
\hline Skull & & & \\
\hline Outer table & 7300 & 0.22 & 3000 \\
\hline Dipole & 3400 & 0.22 & 1744 \\
\hline Inner table & 7300 & 0.22 & 3000 \\
\hline CSF & 2.19 & 0.489 & 1040 \\
\hline Brain & 2190 & 0.4996 & 1040 \\
\hline
\end{tabular}

Table 2. Young modulus, density and poison's ratio of the head [3]

\begin{tabular}{|l|l|l|l|l|}
\hline & Skull 1 & Skull 2 & Skull 3 & Skull 4 \\
\hline Volume $(\mathrm{mm} 3)$ & 663796.71 & 616368.55 & 687490.09 & 585843.68 \\
\hline Surface $(\mathrm{mm} 2)$ & 274159.51 & 263806.94 & 267584.24 & 249803.79 \\
\hline Thickness $(\mathrm{mm})$ & 11.2 & 10.36 & 12.18 & 8.64 \\
\hline Triangles & 286772 & 268898 & 282116 & 238052 \\
\hline
\end{tabular}

Table 3. Volumes, surfaces and number of elements (triangles) of four skulls

Elastic properties were assigned to brain. As per the [3, 35] viscoelastic or elastic properties do not make any fundamental change to the FEM response. Boundary condition details were provided in Figure 14. 


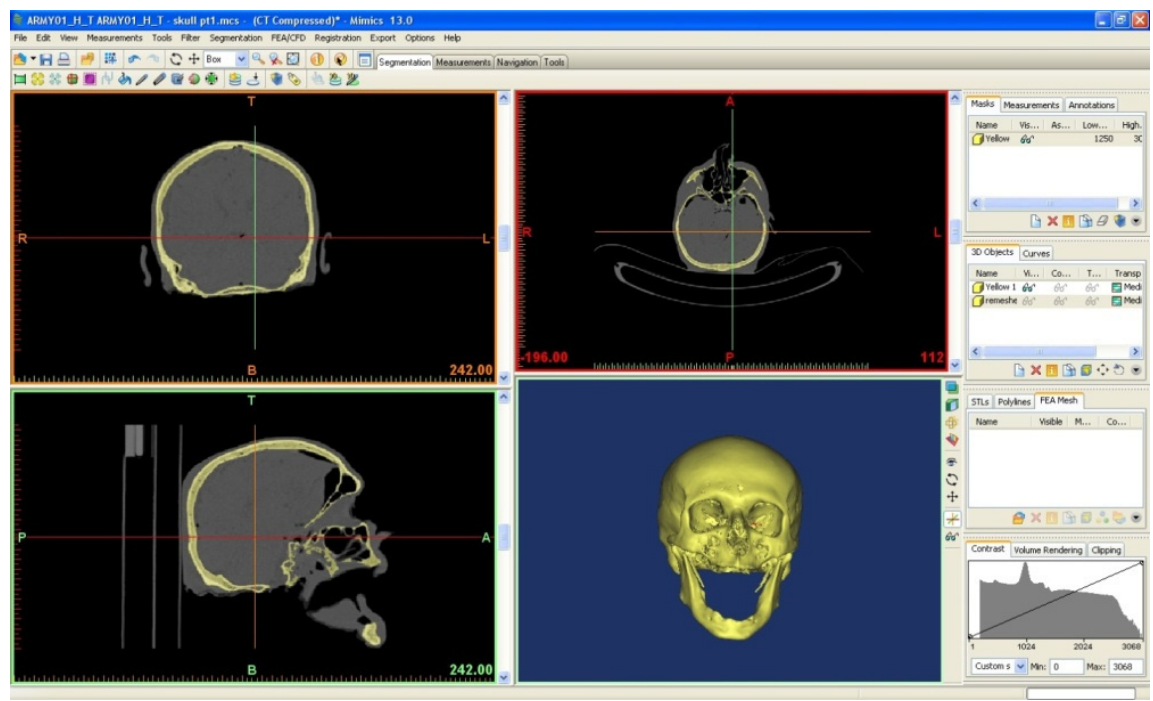

Figure 12. Use of Mimics to create 3D models of human head
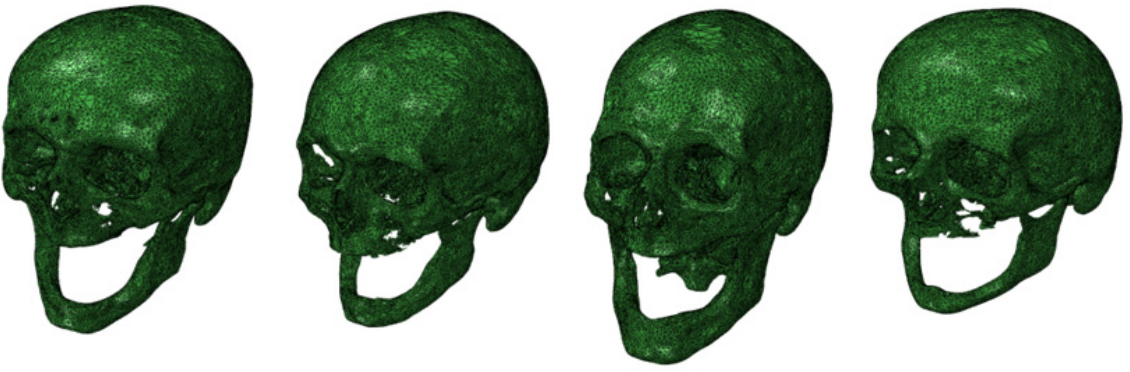

Figure 13. Four meshed skulls in ABAQUS
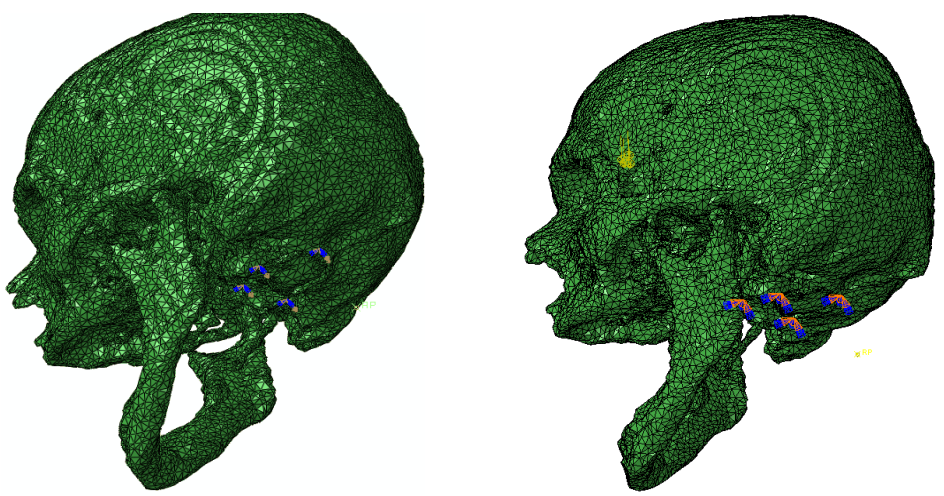

Figure 14. Blue/purple markers show the spots on at neck-head junction where boundary conditions were applied 
As shown in the fig., boundary conditions were defined at the four points around the headneck junction to restrict all transactional movement. Short duration impacts $(<6 \mathrm{~ms})$, the neck does not influence the kinematic head response [35].

\subsection{Validation}

Validation of the model with experimental data was carried out while keeping the properties and load applications same. In order to reproduce the impact conditions, $\sim 8000 \mathrm{kN}$ load was applied to the frontal side of the head, same as in Nahum's experiment [42]. Figure 15 shows pulse duration was kept $2 \mathrm{~ms}$ to reduce the time step cycles. Also, to compare the results for skull fracture with the prior experimental data [34], 8kN-16kN loads were applied.

\subsubsection{Simulation versus experiment}

To simulate the lateral impact, except the impact side on the head, all the other parameters were kept same, load was applied on the lateral side (left side) of the head as shown in Figure 14 .

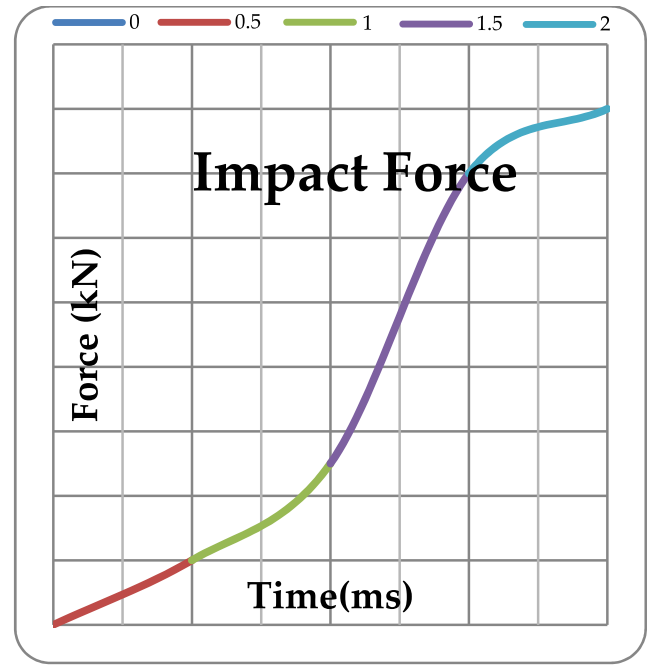

Nahum Experiment [42, 2]

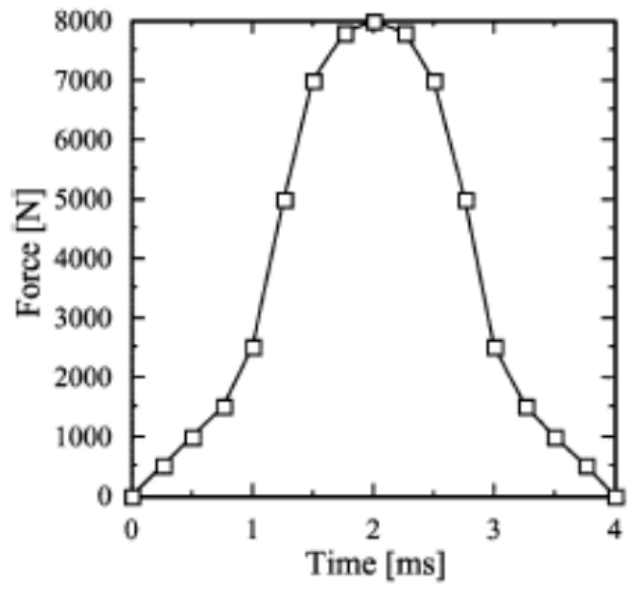

This simulation

Figure 15. Comparison of impact force- time curve between Nahum's experiment and current simulation

\section{Results and discussion}

The frontal impact on the head model predicted the same pressure on coup side as predicted in Nahum's Experiment [42]. This result validates the calibration runs as shown in Figure 16. The model duplicated the experimental response reasonably well, the only minor 
differences attributable to one or more of the following factors: the mesh fineness, reduced frame time steps, or by the material properties. An autopsy did not reveal any visible injury as a result of the Nahum experimental test and, therefore, based on this observation the brain tolerance thresholds were: compression: $234 \mathrm{kPa}$, tension: $186 \mathrm{kPa}$.

A $16 \mathrm{kN}$ load applied to the frontal side of the head while other parameters kept same. Analysis ran 1.1E-3 seconds due to large number of damaged volumes created after that instance. This was consistent with the Yoganandan [19] and Allsop [20] that fracture occurs because of applied force range of 8.8-17 kN. The intracranial pressure reached $200 \mathrm{kPa}$ which was an indicator for brain contusion, oedema, and haematoma, but the pressure exceeded $200 \mathrm{kPa}$ and reached $249 \mathrm{kPa}$, which was only slightly higher than the threshold limit of brain $(234 \mathrm{kPa})$, see Figure 17.

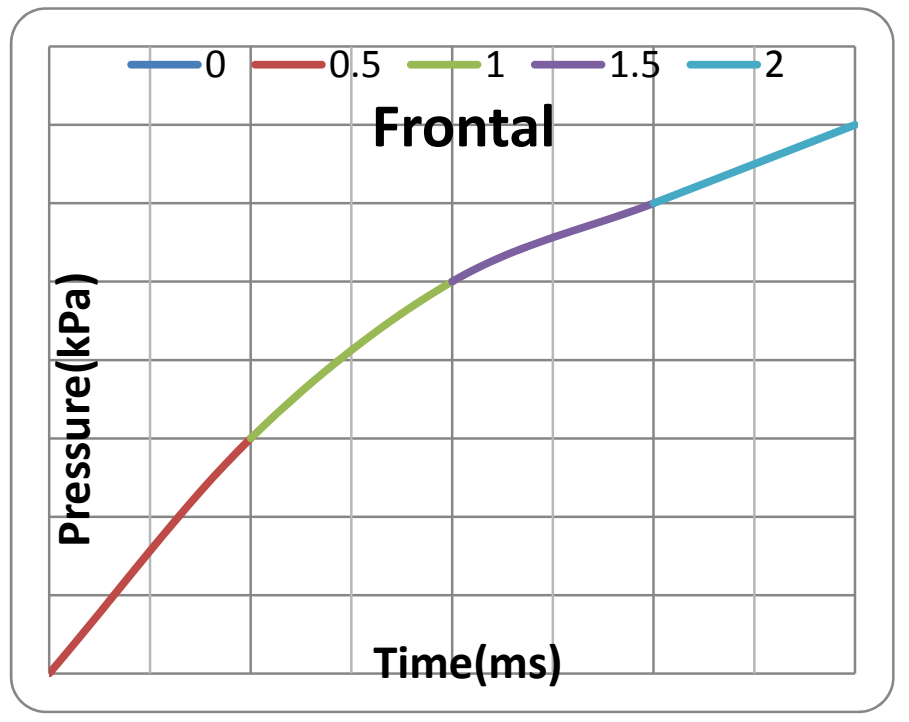

Figure 16. Frontal pressure- time curve results for comparison with Nahum's experimental results.

The history output of strain energy of the model also seemed to be at $2.2 \mathrm{~J}$ consistent with indications of skull fracture Figure 17. Also, from Newton's second law, the resultant acceleration of head can be calculated as $a=16 \mathrm{kN} / 4.5 \mathrm{~kg}$ (sample of patients were of male adults in the age range of 30-50 and the mass of head was considered nearly $\sim 4.5 \mathrm{~kg}$ ).

A fall resulting in head acceleration of over $200 \mathrm{~g}$ and pulse duration of $3.5 \mathrm{~ms}$ or less would create conditions necessary for the production of bridging vein ASDH [4, 28]. Also, a= $355.5 \mathrm{~g}$ is $>150 \mathrm{~g}$ represents the HIC $>2000$ which is non-survival head injury. Thus, these results depict that the model is valid for the further analysis in injury biomechanics. 

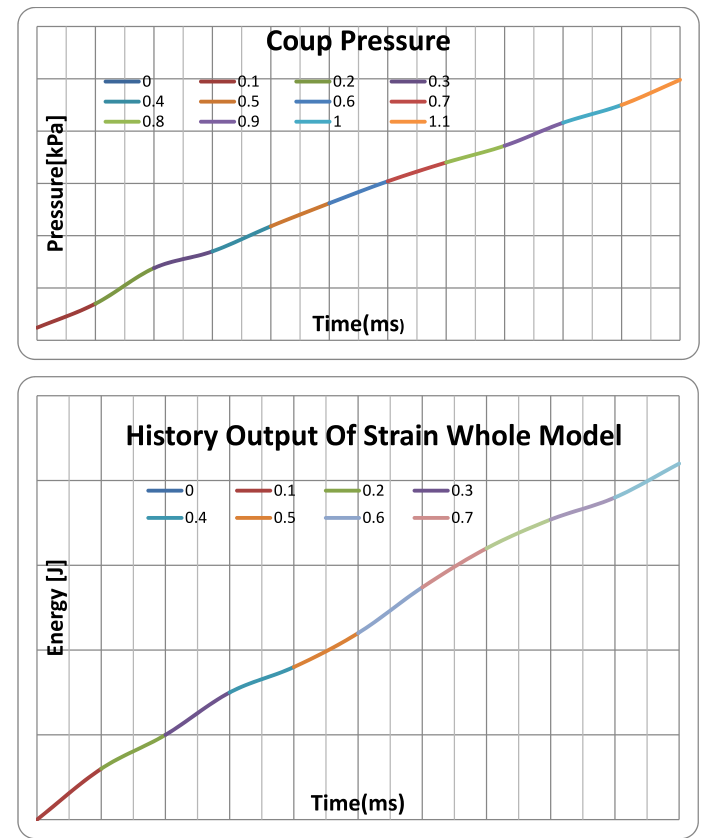

Figure 17. Frontal pressure- time curve and history output of whole strain model after applying 16kN
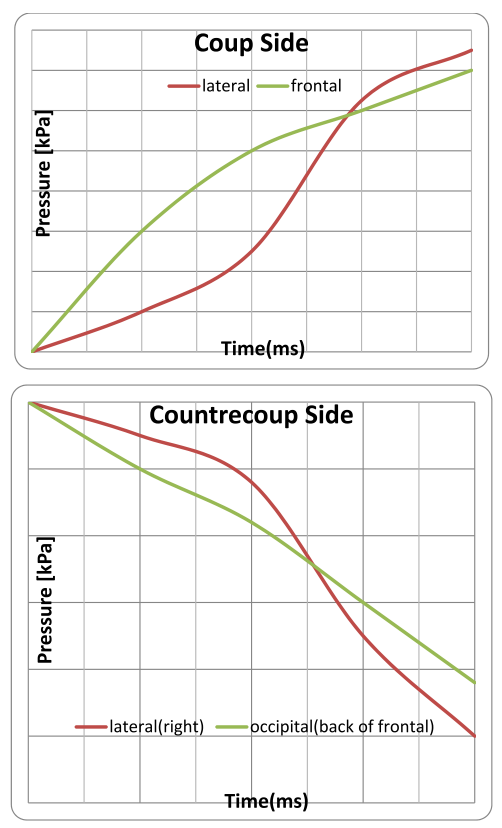

Figure 18. Comparison of pressure-time curves at coup and countercoup sides between lateral and frontal impact 
Simulation result shows that the relative risk and severity of TBI in lateral impacts are higher than in the frontal impacts. Figure 18 shows the pressure-time history for coup and countercoup (at and opposite side of the impact, respectively) sides of the model. It shows quite similar pressure-time curve compared to frontal one. However, the lateral impact produces $6.67 \%$ more pressure at coup side as compared to frontal impact. The results of countercoup side support the prior analysis predicting only $14 \%$ higher tensile stress by lateral as compared to frontal impacts.

Statistical analysis carried out on 1115 occupants who were the victims of lateral and nonlateral automobile impacts [40-41], TBI occurred from lateral impacts were more severe than those resulting from non-lateral impacts.

\section{Conclusion}

The paper reviewed the head injury mechanisms and criteria. A computational framework was developed to biomechanical parameters to assess the injury, and validate the finite element models of the human head. The comparison of the stress/pressure incurred by lateral and frontal impacts in the coup and countercoup side of the head was presented. The model has been validated against the two sets of experimental results: one obtained in frontal impact and the other using head tolerance/skull fracture data.

Although the results obtained from the study involved a degree of inaccurateness (i.e., model had around 6500 distorted elements, 3 layers of skull was assigned as a one layer having the mechanical property (young's modulus, poison's ratio and density) as an average of those 3 layers), they do nonetheless confirm that through proper sets of MRI data, analytical modeling is applicable in injury biomechanics.

It is concluded that the lateral impacts are more severe than the frontal impacts. Therefore, it is imperative that victims of lateral impacts are at more risk for TBI than the frontal impacts. This information may be useful in injury assessment and developing sensors to alleviate lateral impacts to prevent traumatic brain injuries.

\section{Author details}

Aalap Patel and Tarun Goswami*

Department of Biomedical, Industrial and Human Factors Engineering, Wright State University, Dayton, OH, USA

\section{References}

[1] Marjoux, D., Baumgartner, D., Deck, C., and Willinger, R., Head injury prediction capability of the HIC, HIP, SIMon and ULP criteria -New injury criteria for the head. Accident Analysis and Prevention 40(3), 1135-1148, 2008.

\footnotetext{
${ }^{*}$ Corresponding Author
} 
[2] Gilchrist, M. and O'Donoghue, D., 2000, Simulation of the Development of Frontal Head Impact Injury, Comp. Mech., 26, 229-235.

[3] Zong*, Z, H P Lee and C Lu, "A three-dimensional human head finite element model and power flow in a human head subject to impact loading". JOURNAL OF BIOMECHANICS, 39, no. 1 (2006): 284-292. (United Kingdom).

[4] O'Riordain K, Thomas PM, Phillips JP, Gilchrist MD. Reconstruction of real world head injury accidents resulting from falls using multibody dynamics. Clin Biomech 2003; 18:590-600.

[5] E. A. C. Johnson and P. G. Young, "On the use of a patient-specific rapid-prototyped model to simulate the response of the human head to impact and comparison with analytical and finite element models," J. Biomechan., vol. 38, pp. 39-45, 2005.

[6] Shi Wei Gong, Heow Pueh Lee, Chun Lu., Dynamic response of a human head to a foreign object impact, Inst. of High Performance Comput., Singapore;. Biomedical Engineering, IEEE Transactions on. 04/2008; 55(3):1226-1229

[7] Shorten M.R. \& Himmelsbach, J.A. (2003),Sports surfaces and the risk of traumatic brain injury, pp 49-69 in sports surfaces(Eds. B.M. Nigg, G.K. Cole, D.J. Stefanyshyn)Calgary, University of Calgary

[8] http://www.pas.rochester.edu/ blackman/ur10helmetsimpact.pdf

[9] Portions from Pike, J. A., Automotive Safety: Anatomy, Injury, Testing and Regulation, SAE, 1990

[10] http://homepages.nyu.edu/ eh597/Meninges.htm

[11] "Head Injury Criterion and the ATB", Presented at the 2004 ATB Users' Conference, Salt Lake City, UT (http://www.mchenrysoftware.com/HIC\%20and\%20the\%20ATB.pdf)

[12] Copyright @ 2001 - 2011 BrainInjury.com / Steven (Woody) Igou

[13] http://www.peoplecentric.info/tbi-autism-aspeger-assocations/

[14] Ommaya, A.K., Thibault, L., Bandak, F.A., 1994. Mechanisms of impact head injury. Int. J. Impact Eng. 15, 535-560.

[15] Jennett, B., 1996. Epidemiology of head injury. J. Neurol. Neurosurg. Psychiatry 60, 362369.

[16] S. W. Gong, H. P. H. P. Lee, and C. Lu, "An approach for the estimation of contact force on a human head induced by a foreign-object impact," IEEE Trans. Biomed. Eng., vol. 54, no. 5, pp. 956-958, May 2007.

[17] Young, P.G., 2003. An analytical model to predict the response of fluid-filled shells to impact-a model for blunt head impacts. Journal of Sound and Vibration 267, 11071126.

[18] J. S. Ruan, T. B. Khalil, and A. I. King, "Dynamic response of the human head to impact by three-dimensional finite element analysis," J. Biomechan. Eng., vol. 116, pp. 44-50, 1994.

[19] Yoganandan, N., Pintar, F.A., Sances Jr., A., Walsh, P.R., Ewing, C.L., Thomas, D.J., Snyder, R.G., 1995. Biomechanics of skull fractures. J. Neurotrauma 12, 659-668.

[20] Allsop, D.L., Perl, T.R., Warner, C.Y., 1991. Force/deflection and fracture characteristics of the temporo-parietal region of the human head. In: Proc. 35th Stapp Car Crash Conf., pp. 269-278. 
[21] Gurdjian ES, Webster JE, 1945. Linear acceleration causing shear in the brainstem in trauma of the central nervous system. Mental Adv Dis 24:28.

[22] Gurdjian ES, Webster JE, Lissner HR, 1955. Observations on the mechanism of brain concussion, contusion and laceration. Surg Gynec Obstet 101:680-690.

[23] Lissner HR, Lebow, M, Evans FG, 1960. Experimental studies on the relation between acceleration and intracranial changes in man. Surg Gynecol Obstet 11: 329-338.

[24] Patrick LM, Lissner HR, Gurdjian ES, 1963. Survival by design - head protection. Proc 7th Stapp Car Crash Conference 36: 483-499.

[25] Gadd CW, 1966. Use of a weighted impulse criterion for estimating injury hazard. Proc 10th Stapp Car Crash Conference; SAE Paper 660793, Society of Automotive Engineers, Warrendale PA, USA.

[26] National Highway Traffic Safety Administration (NHTSA), Department of Transportation, 1997.FMVSS201, Head Impact Protection, 49 CFR §571.201.

[27] Prasad P, Mertz HJ, 1985. The position of the United States delegation to the ISO working group on the use of HIC in the automotive environment. SAE Paper\# 851246 Society of Automotive Engineers, Warrendale PA, USA.

[28] Gennarelli, T.A., Thibault, L.E., 1982. Biomechanics of acute subdural hematoma. J. Trauma 22, 680-686.

[29] Ryan, G.A., Vilenius, A.T.S., 1994. Field and analytic observations of impact brain injury in fatally injured pedestrians. In: Proceedings of the Head Injury_94 Conference, Washington, DC sponsored by US National Highway Traffic Safety Administration and George Washington University, 12-14 October, pp. 181-188.

[30] Enouen, S.W., 1986. Development of experimental head impact procedures for simulating pedestrian head injury. In: Proc. 30 ${ }^{\text {th }}$ Stapp Car Crash Conf., pp. 199-218.

[31] MacLaughlin, T.F., Wiechel, J.F., Guenther, D.A., 1993. Head impact reconstructionHIC validation and pedestrian injury risk. In: Proc. of the SAE Conf. on Accident Reconstruction: Technology and Animation III, pp. 175-183

[32] Mohan, D., Bowman, B.M., Snyder, R.G., Foust, D.R., 1979. A biomechanical analysis of head impact injuries to children. J. Biomech. Eng. 101, 250-260.

[33] Auer, C., Schonpflug, M., Beier, G., Eisenmenger, W., 2001. An analysis of brain injuries in real world pedestrian traffic accidents by computer simulation reconstruction. In: Proc. Int. Soc. Biomechanics XVIIIth Congress.

[34] Doorly MC, Gilchrist MD. The use of accident reconstruction for the analysis of traumatic brain injury due to head impacts arising from falls. Computational Methods in Biomechanics and Biomedical Engineering. 2006;9:371-377

[35] Remy Willinger, Ho-Sung Kang, and Baye Diaw, “Three-Dimensional Human Head Finite Element Model Validation against Two Experimental Impacts", Annals of Biomedical Engineering, Vol. 27, pp. 403-410, 1999.

[36] D. Baumgartner ${ }^{\mathrm{a}}$, R. Willingera, J.S. RAUL ${ }^{\mathrm{b}}$ Finite element modeling of the human head and application to forensic medicine, 19ème Congrès Français de Mécanique, Marseille, 2428 août 2009 
[37] Baumgartner D., Willinger R., Human head tolerance limits to specific injury mechanisms inferred from real world accident numerical reconstruction, Revue Européenne des Eléments Finis, vol. 14, n 4-5, pp. 421-444, 2005.

[38] Ward, C. C., M. Chan, and A. M. Nahum. Intracranial pressure-A brain injury criterion. In: Proceedings of the 24th Stapp Car Crash Conference, SAE Paper No. 801304, 1980, pp. 347-360.

[39] Bazarian JJ, Fisher SG, Flesher W, Lillis R, Knox KL, Pearson TA., Lateral automobile impacts and the risk of TBI, Ann Emerg Med. 2004 Aug;44(2):142-52.

[40] Morris A, Hassan A, Mackay M, et al. Head injuries in lateral impact collisions. Accid Anal Prev. 1995;27:749-756.

[41] McLellan BA, Rizoli SB, Brenneman FD, et al. Injury pattern and severity in lateral motor vehicle collisions: a Canadian experience. J Trauma. 1996;41:708-713

[42] Nahum, A. M., R. Smith, and C. C. Ward. Intracranial pressure dynamics during head impact. In: Proceedings of the 21st Stapp Car Crash Conference, 1977, pp. 339-366.

[43] Willinger, R., L. Taleb, and C. M. Kopp. Modal and temporal analysis of head mathematical models. J. Neurotrauma 12:N4, 1995.

[44] http://www.materialise.com/mimics

[45] ABAQUS Dynamic Explicit Version 6.9 Hibbitt, Karlsson\& Sorenson,Inc., Pawtucket, RI

[46] http://www.eurailsafe.net/subsites/operas/HTML/appendix/Table13.htm

[47] http://www.eurailsafe.net/subsites/operas/HTML/Section3/Page3.3.1.4.htm

[48] Jason Kerrigan1, Carlos Arregui2, Jeff Crandall1,Pedestrian head impact dynamicscomparison of dummy and PHMS IN SMALL SEDAN AND LARGE SUV IMPACTS (www-nrd.nhtsa.dot.gov/pdf/esv/esv21/09-0127.pdf) 